\title{
Lactotransferrin expression is downregulated and affects the mitogen-activated protein kinase pathway in gastric cancer
}

\author{
GENGQIU LUO $^{1 *}$, YANHONG ZHOU $^{2 *}$, WEI YI ${ }^{2}$ and HONG YI ${ }^{3}$ \\ ${ }^{1}$ Department of Pathology, Xiangya Hospital, Central South University, Changsha, Hunan 410008; \\ ${ }^{2}$ Molecular Genetics Laboratory, Cancer Research Institute, Central South University, Changsha, Hunan 410078; \\ ${ }^{3}$ Research Center of Carcinogenesis and Targeted Therapy, Xiangya Hospital, \\ Central South University, Changsha, Hunan 410008, P.R. China
}

Received July 12, 2014; Accepted February 17, 2015

DOI: $10.3892 / \mathrm{ol} .2015 .3011$

\begin{abstract}
Gastric cancer (GC) is the second leading cause of cancer-associated mortality worldwide. In advanced and metastatic GC, conventional chemotherapy results in limited efficacy and the average survival rate is currently approximately 10 months. Dysregulated activation of numerous genes, including zinc finger, DHHC-type containing 14; caspase-associated recruitment domain-containing protein; and Ras association domain family member 10 , have been implicated in GC. The tumor suppressor function of lactotransferrin (LTF) has been reported in a variety of tumors, including $\mathrm{GC}$, nasopharyngeal carcinoma (NPC) and prostate cancer. However, the mechanism of the tumor suppressor function of LTF in GC remains unclear. In the present study, the expression levels of LTF in patient GC tissue samples were investigated using reverse transcription-quantitative polymerase chain reaction, and it was demonstrated that the LTF mRNA expression level in GC tissue samples was reduced by 20 -fold compared with the adjacent non-cancerous tissues $(t=4.56, \mathrm{P}<0.01)$. A similar trend in LTF protein expression was observed by western blot analysis. Furthermore, the present study demonstrated that the mitogen-activated protein kinase (MAPK) signaling pathway intermediates p38, $c$-Jun N-terminal kinase (JNK) and $c$-Jun were highly expressed in GC tissue samples,
\end{abstract}

Correspondence to: Professor Hong Yi, Research Center of Carcinogenesis and Targeted Therapy, Xiangya Hospital, Central South University, 87 Xiangya Road, Changsha, Hunan 410008, P.R. China

E-mail: yihongxy2014@163.com

*Contributed equally

Abbreviations: GC, gastric cancer; LTF, lactotransferrin; JNK, $c$-Jun $\mathrm{N}$-terminal kinase; MAPK, mitogen-activated protein kinase; TP53, tumor protein p53; GADPH, glyceraldehyde-3-phosphate dehydrogenase

Key words: mitogen-activated protein kinase, lactotransferrin, gastric cancer, expression and indicated that LTF downregulation may be associated with the dysregulation of the MAPK signaling pathway in GC tissues. In addition, the present study indicated that LTF overexpression reduced the expression of p38, JNK2 and $c$-Jun in the GC cell line, SGC7901. The present study demonstrates that LTF expression is downregulated in GC tissues and that LTF may serve an important role in the dysregulation of the MAPK signaling pathway.

\section{Introduction}

Gastric cancer (GC) is one of the most common types of malignant cancer, with poor prognosis and high mortality rates worldwide $(1,2)$. Therefore, the development of an effective therapeutic method with minimal side effects is required. Early diagnosis and curative resection are associated with increased survival in patients with GC. However, currently the majority of GC cases are diagnosed at later stages. The generation of differential expression profiles from pathological samples can markedly improve cancer biomarker discovery (2). In a previous study, it was demonstrated that Sox 7 and $\beta$-catenin expression significantly correlated with the depth of invasion, lymph node metastasis, distant metastasis and the TNM (tumor, node and metastasis) stage in GC (3). Overexpression of zinc finger, DHHC-type containing 14 (ZDHHC14) promotes the migration and invasion of scirrhous GC (4). Caspase-associated recruitment domain-containing protein (CARP) is a potential tumor suppressor in GC, and a single-nucleotide polymorphism in the CARP gene may increase the risk of GC (5). Ras association domain family member 10 (RASSF10) is an epigenetically silenced tumor suppressor in GC (6). It has previously been demonstrated that the inactivation of tumor suppressor genes and activation of oncogenes serve a significant role in carcinogenesis (3-6). However, the etiology of GC remains poorly understood.

Lactotransferrin (LTF; also termed lactoferrin) is an iron-binding glycoprotein involved in a large array of protective processes in mammals, resulting in antibacterial, antioxidant and anticarcinogenic effects for the host (7-9). LTF is produced in the exocrine glands and is secreted in numerous external fluids as a first line of defense (9). LTF has the capacity to induce apoptosis and inhibit proliferation in cancer cells, and restore 
white and red blood cell levels following chemotherapy (9). The tumor suppressor function of LTF has been reported in a variety of tumors (10-15), however the main functions of LTF are within innate immunity and nutrition $(7,8)$. In a previous study, LTF was observed to inhibit the cell migration of three gastrointestinal cell lines (Caco-2, AGS and IEC-18) in vitro (16). Other studies have examined the association between LTF and GC, but the underlying mechanism remains unclear.

The MAPK pathway is an important signaling pathway in many malignancies. We previously found that lactotransferrin, a candidate tumor suppressor, exhibited deficient expression in human nasopharyngeal carcinoma and inhibited nasopharyngeal carcinoma cell proliferation by modulating the mitogen-activated protein kinase pathway (17) Therefore, in the present study, the expression levels of LTF and the key molecular intermediates of the MAPK signaling pathway [p38, $c$-Jun N-terminal kinase (JNK) and $c$-Jun] were investigated in GC tissues. In addition, the effects of overexpressing LTF were studied in the GC cell line, SGC7901.

\section{Materials and methods}

Cell culture. The human SGC7901 GC cell line was cultured in RPMI 1640 (Gibco Life Technologies, Carlsbad, CA, USA) supplemented with $10 \%$ fetal bovine serum (Gibco Life Technologies), $100 \mathrm{U} / \mathrm{ml}$ penicillin and $100 \mu \mathrm{g} / \mathrm{ml}$ streptomycin (GE Healthcare Life Sciences, Logan, UT, USA) at $37^{\circ} \mathrm{C}$ in the presence of $5 \% \mathrm{CO}_{2}$.

Patient samples. A total of 8 participants were recruited at Xiangya Hospital, Central South University (Changsha, China). Consent forms were obtained from individual patients and experimental protocols were approved by the Institutional Review Board of Xiangya Hospital (Changsha, China). All subjects enrolled in the study were Chinese. All clinical and biological data available for the samples are listed in Table I. GC and corresponding non-tumor tissues were collected and each biopsy sample was divided into two sections; one was submitted for routine histological diagnosis and the remaining section was assessed by quantitative polymerase chain reaction (qPCR) and western blot analysis.

$R N A$ isolation and reverse transcription (RT)-qPCR analysis. Total RNA was extracted using a TRIzol reagent (Invitrogen Life Technologies, Carlsbad, CA, USA). The quality of RNA samples was determined by $1 \%$ agarose (Invitrogen Life Technologies) gel electrophoresis at $80 \mathrm{~V}$ for 40 min followed by $60 \mathrm{~min}$ at $120 \mathrm{~V}$, and staining with SYBR Safe DNA Gel Stain kit (Invitrogen Life Technologies). Bands of $18 \mathrm{~S}$ and 28S RNA were visualized under UV light (multi-purpose ultraviolet analyzer, model:WD-9403F; Beijing Liuyi Instrument Factory,Beijing, China). The optical density 260/280 ratios of the samples were demonstrated to be in the range of 1.8-2.0. The chromosomal DNA was removed from the total RNA preparation by using RQ1 RNase-Free DNase (Promega Corporation, Beijing, China). For detection of LTF mRNA expression levels, cDNA was synthesized from total RNA using a Reverse Transcription System (Promega Corporation) according to the manufacturer's instructions; GAPDH was used as an internal control. The sequences of the primers used for the qPCR were
Table I. Characteristics of patients with gastric cancer.

\begin{tabular}{lccc}
\hline Samples & Gender & Age & Histological diagnosis \\
\hline a & Male & 69 & Well differentiated \\
b & Female & 63 & Intermediately differentiated \\
c & Male & 49 & Poorly differentiated \\
d & Male & 60 & Intermediately differentiated \\
e & Male & 73 & Poorly differentiated \\
f & Male & 62 & Poorly differentiated \\
g & Female & 61 & Intermediately differentiated \\
h & Male & 58 & Poorly differentiated \\
\hline
\end{tabular}

as follows: LTF, F 5'-GCATGGGCTAAGGATTTGAA-3' and R 5'-TCCCAAATTTAGCCTGTTGG-3'; GAPDH, F 5'-CGA CCACTT TGTCAAGCTCA-3' and R 5'-ACTGAGTGT GGCAGGGACTC-3'. The expression levels of mRNA were assessed by evaluating the relative threshold cycle $(\mathrm{Ct})$ values. The $\mathrm{Ct}$ values were normalized against the expression levels of GAPDH, and the relative amount of mRNA specific to each of the target genes was calculated using the $\Delta \Delta C T$ method (17-19).

Construction of the pIRES-LTF vector. The full-length LTF coding sequence entirely lacking the 3'-UTR (GeneCopoeia, Rockville, MD, USA) was subcloned into the eukaryotic expression vector pIRES (Clontech Laboratories, Inc., Mountainview, CA, USA) as previously described (20). The fusion sequences were verified by DNA sequencing using a Sanger ABI 3730x1 DNA sequencer (GATC Biotech AG, Konstanz, Germany). The empty pIRES vector was used as a negative control.

Cell transfection. Cell transfection was performed using Lipofectamine (Invitrogen Life Technologies), according to the manufacturer's protocol. Cells were seeded into each well of a 6-well plate, at a density of $2 \times 10^{5}$ cells/well, $24 \mathrm{~h}$ prior to transfection. For transfection, $2 \mu \mathrm{g}$ each of the pIRES-LTF and pIRES plasmids were transfected into the SGC7901 cells. The plasmids were diluted with $100 \mu \mathrm{l}$ serum-free media and $4 \mu \mathrm{l}$ Lipofectamine was added into $100 \mu \mathrm{l}$ serum-free media. The two solutions were combined, mixed gently and incubated at room temperature for $30 \mathrm{~min}$. Next, the $200 \mu \mathrm{l}$ mixture and a further $200 \mu \mathrm{l}$ serum-free media were added into each well. The cells were then incubated at $37^{\circ} \mathrm{C}$ for $24 \mathrm{~h}$, followed by replacing the transfection media with fresh complete culture media. Following an additional 48-h culture, the cells were harvested for the following western blot analysis.

Western blot analysis. The GC tissues, corresponding non-tumor tissues and SGC7901 cells were lysed in RIPA buffer (Beijing Comwin Biotech Co., Ltd, Beijing, China) and the total protein concentration was determined using a Pierce BCA Protein Assay Kit (Thermo Fisher Scientific, Inc., Rockford, IL USA). Extracts containing $50 \mu \mathrm{g}$ protein were electrophoretically separated on 10\% SDS-PAGE gels and electroblotted onto nitrocellulose membranes (GE Healthcare Life Sciences, Logan, UT, USA). The membranes were blocked using Tris-buffered saline/Tween $20(25 \mathrm{mM}$ Tris-HCl, $150 \mathrm{mM} \mathrm{NaCl}, \mathrm{pH} 7.5$ and $0.05 \%$ Tween 20) containing 
A

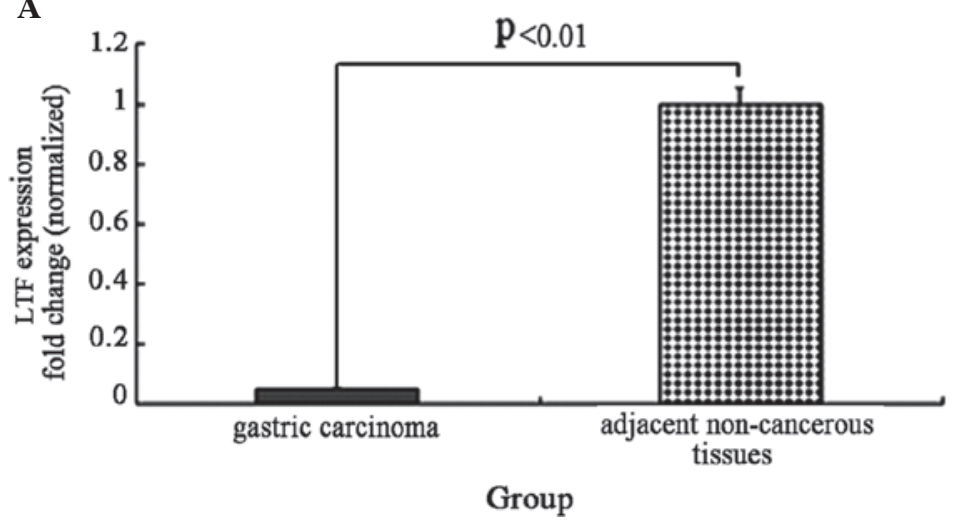

B

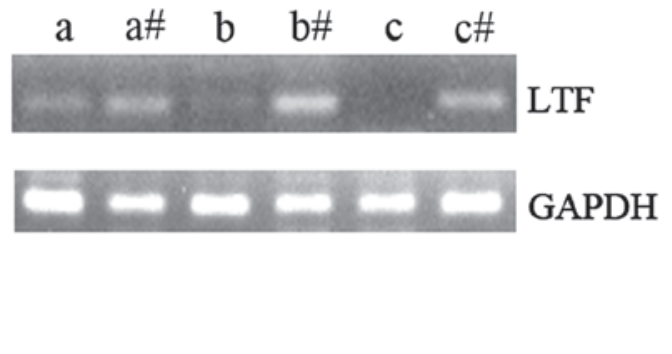

Figure 1. Differential expression of the LTF gene in gastric cancer and adjacent non-cancerous tissues assessed by quantitative polymerase chain reaction. (A) Normalized LTF gene expression in gastric cancer was 20 -fold compared with adjacent non-cancerous tissues ( $\mathrm{n}=8$ ). (B) Agarose gel electrophoresis images present LTF and GAPDH gene expression in three gastric cancer samples and adjacent non-cancerous tissues. Gastric cancer tissues: a, b and c; adjacent non-cancerous tissues: a\#, b\# and c\#. Data are representative of three independent experiments. LTF, lactotransferrin.

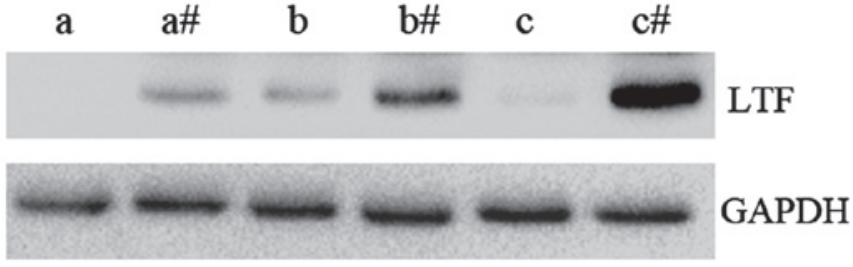

Figure 2. Expression levels of LTF protein in gastric cancer and adjacent non-cancerous tissues assessed by western blot analysis using the same tissues as assessed in Fig. 1. Gastric cancer tissues: a, b and c; adjacent non-cancerous tissues: a\#, b\# and c\#. Data are representative of three independent experiments. LTF, lactotransferrin.

$5 \%$ non-fat milk followed by an overnight incubation at $4^{\circ} \mathrm{C}$ with the following primary antibodies: Rabbit anti-human p38 antibody (1:200; catalog no. B7178; Anbo Biotechnology Company, Changzhou, China); rabbit anti-human JNK2 antibody (catalog no. sc-827) and rabbit anti-human $c$-Jun antibody (1:500; catalog no. sc-1694; Santa Cruz Biotechnology, Inc., Santa Cruz, CA, USA); and rabbit anti-human p53 antibody (1:200; catalog no. PB0076; Wuhan Boster Biological Technology, Ltd., Wuhan, China). Following three washes with phosphate buffered saline with Tween (Beyotime Institute of Biotechnology, Hangzhou, China), the membranes were incubated with horseradish peroxidase-conjugated secondary antibodies (Santa Cruz Biotechnology, Inc.) and the specific signals were visualized using an ECL detection system [MultiSciences (Lianke) Biotech Co., Ltd. (Hangzhou, China]. Anti-human GAPDH antibody (1:3,000; Santa Cruz Biotechnology, Inc.) was used as a loading control.

Statistical analysis. Data are expressed as the mean \pm standard deviation. Differences of the quantitative variables between groups were analyzed by Student's t-test using SPSS software, version 11.0 (SPSS, Inc., Chicago, IL, USA). $\mathrm{P}<0.05$ was considered to indicate a statistically significant difference.

\section{Results}

Detection of $m R N A$ expression levels of LTF in GC. To detect the mRNA expression levels of the LTF gene in GC and adjacent non-cancerous tissues, eight samples of each were collected and subjected to RT-qPCR to examine the LTF gene. The data were analyzed using the $\Delta \Delta \mathrm{CT}$ method and the fold change in the expression of LTF was calculated relative to the internal control gene, GAPDH. The expression of the LTF gene was lower in the GC samples compared with the adjacent non-cancerous tissues, and the normalized LTF gene expression in gastric cancer tissue samples was reduced by $\sim 20$-fold $(\mathrm{t}=4.56, \mathrm{P}<0.01$; Fig. 1A). Agarose gel electrophoresis was performed following RT-qPCR to assess the LTF and GAPDH genes in three GC tissues and adjacent non-cancerous tissues (Fig. 1B). The results from the electrophoresis further demonstrate that LTF expression is deficient in GC tissues compared with adjacent non-cancerous tissues.

Western blot analysis of protein levels of LTF in GC. The protein expression levels of LTF were examined in GC samples and compared to adjacent non-cancerous tissues (Fig. 2). In agreement with the qPCR results, the expression level of LTF protein was observed to be lower in GC tissues compared with the adjacent non-cancerous tissues. These results further demonstrate that LTF expression levels are downregulated in GC.

LTF correlates with dysregulation of the MAPK signaling pathway in GC tissues. In order to identify the possible underlying mechanism of LTF action in GC, the expression levels of key intermediate molecules in the MAPK signaling pathway were assessed by western blot analysis. p38, JNK2 and $c$-Jun were upregulated in GC tissues compared with the adjacent non-cancerous tissues (Fig. 3). In addition, the expression levels of p53 protein were markedly downregulated in GC tissues compared with the adjacent non-cancerous tissues (Fig. 3). The results of the present study indicate that LTF may be associated with the dysregulation of the MAPK signaling pathway in GC tissues. These results may indicate an association between reduced LTF expression levels and the upregulation of certain key molecular intermediates of the MAPK signaling pathway in GC.

LTF overexpression affects the expression of $p 38, J N K 2$ and $c$-Jun in vitro. To confirm whether LTF affected the expression 


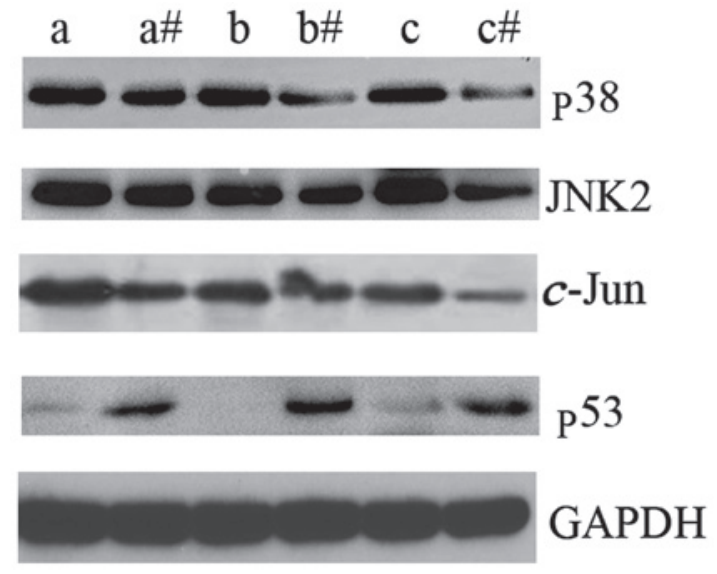

Figure 3. Expression levels of p38, JNK2, $c$-Jun and p53 protein in gastric cancer and adjacent non-cancerous tissues assessed by western blot analysis using the same tissues as assessed in Fig. 1. Gastric cancer tissues: a, b and c; adjacent non-cancerous tissues: a\#, b\# and c\#. Data are representative of three independent experiments. JNK, $c$-Jun N-terminal kinase.

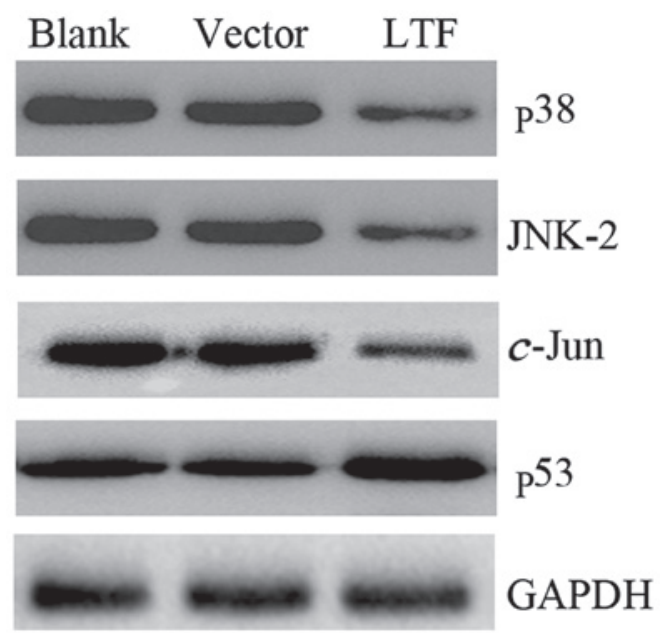

Figure 4. Protein expression levels of p38, JNK2, $c$-Jun and p53 in the gastric cancer cell line, SGC7901. LTF, SGC7901 cells transfected with pIRES-LTF plasmid; vector, SGC7901 cells transfected with pIRES plasmid; blank untransfected SGC7901 cells. Data are representative of three independent experiments. JNK, $c$-Jun N-terminal kinase; LTF, lactotransferrin.

of p38, JNK2 and $c$-Jun in vitro, pIRES-LTF and pIRES were transfected into the SGC7901 GC cell line. The cells were harvested $48 \mathrm{~h}$ following transfection and the levels of the p38, JNK2, $c$-Jun and p53 proteins were determined. The results demonstrated that $\mathrm{p} 38, \mathrm{JNK} 2$ and $c$-Jun proteins were expressed at lower levels in the SGC7901 cells transfected with pIRES-LTF compared with the SGC7901 cells transfected with pIRES (Fig. 4). However, the p53 protein expression levels displayed the opposite pattern. p53 was highly expressed in the SGC7901 cells transfected with pIRES-LTF compared with the SGC7901 cells transfected with pIRES (Fig. 4). These results demonstrate that LTF overexpression affects the expression of $\mathrm{p} 38, \mathrm{JNK} 2, c$-Jun and $\mathrm{p} 53$ in vitro.

In conclusion, the overexpression of LTF downregulates the expression of p38, JNK2 and $c$-Jun. However, the mechanism by which LTF affects the MAPK signaling pathway requires further investigation.

\section{Discussion}

It has previously been demonstrated in numerous studies that LTF possesses antitumor action $(10,15,21,22)$. In the current study, it was demonstrated that the mRNA and protein expression levels of LTF in GC were 20-fold lower compared with the adjacent non-cancerous tissues. Sousa et al (2) observed significantly lower levels of LTF in GC samples with more advanced disease progression and worse prognosis. In a previous study, quantitative analysis of LTF mRNA expression revealed a marked downregulation in prostate cancer (23). In addition, the LTF gene is inactivated by genetic and epigenetic mechanisms in lung cancer (24). Previous studies have also indicated that LTF may have a direct effect on tumor cell growth, as suggested by the fact that LTF and an LTF splice variant are downregulated or absent in certain types of cancer (25-28). The results of the present study indicate that LTF may also serve as an important tumor suppressor gene in GC.

To explore the possible mechanism of LTF in GC, the expression levels of key signaling intermediates (p38, JNK2 and $c$-Jun) in the MAPK signaling pathway were assessed. Compared with the adjacent non-cancerous tissues, p38, JNK2 and $c$-Jun were upregulated in GC samples. This result may reflect a negative association between the expression of LTF and the key molecular intermediates of the MAPK signaling pathway in GC tissues. In addition, the effect of overexpression of LTF on levels of p38, JNK2 and $c$-Jun was determined in vitro. The results demonstrated that overexpression of LTF downregulated the expression of p38, JNK2 and $c$-Jun in the SGC7901 cells. MAPKs transduce extracellular signals, promoting a variety of cellular processes, including cell proliferation, survival, death and differentiation (29). JNK and p38 MAPK signaling are associated with various types of cancer in humans and mice (30). JNK is a family of protein kinases that are activated by stress stimuli and regulate various cellular processes, including proliferation, apoptosis and survival (31). Yuan et al (32) observed that the overexpression of human DNA polymerase $\iota$ (pol $\iota)$ is positively correlated with clinical tumor grade in bladder cancer samples and may contribute to hypermutagenesis. Dysregulation of pol $\iota$ by $\mathrm{JNK} / c$-Jun is involved in carcinogenesis and offers a novel understanding of the role of pol $\iota$ or $c$-Jun in mutagenesis (32). In A549 human non-small cell lung cancer cells, JNK may be specifically required in vivo for the maintenance of the tumor-initiating population of tumor cells rather than for the proliferation and survival of the entire cell population (33). Taken together, the results of the present study and previous studies indicate that a lack of LTF expression in GC may increase the expression of p38, JNK2 and $c$-Jun.

In addition, the present study demonstrated that p53 was downregulated in GC, and that the overexpression of LTF increased the levels of p53 protein in vivo. p53 is a well-documented tumor suppressor gene (34). The functions of p53 include responding to cellular stress, apoptosis and cell cycle arrest. The p53 pathway is inactivated in the majority of types of human cancer (34-37). MicroRNA hsa-miR-125a-3p activates the p53 protein and induces lung cancer cell apoptosis (35). p53 acts as a safeguard of protein synthesis by regulating the rRNA methyltransferase fibrillarin and the 
subsequent quality and intrinsic activity of ribosomes (36). A previous study demonstrated that the loss of $\mathrm{p} 53$ promotes the invasion and metastasis capability of prostate cancer cells through the FAK-Src signaling pathway (37). The association between LTF, p53 and GC requires further investigation.

In conclusion, the present study demonstrates that LTF expression is downregulated in GC, and that this may affect the MAPK signaling pathway. However, the details of the underlying mechanism require further study.

\section{Acknowledgements}

The present study was supported by the National Natural Science Foundation of China (grant nos. 81272975 and 81172302); the Key Project of Hunan Provincial Natural Science Foundation (grant no. 12JJ2044); the Project of Hunan Provincial Natural Science Foundation (grant no. 12JJ3121); and the Project of Hunan Provincial Development and Reform Commission.

\section{References}

1. Cho JY: Molecular diagnosis for personalized target therapy in gastric cancer. J Gastric Cancer 13: 129-135, 2013.

2. Sousa JF, Ham AJ, Whitwell C, Nam KT, Lee HJ, Yang HK, et al: Proteomic profiling of paraffin-embedded samples identifies metaplasia-specific and early-stage gastric cancer biomarkers Am J Pathol 181: 1560-1572, 2012.

3. Cui J, Xi H, Cai A, Bian S, Wei B and Chen L: Decreased expression of Sox7 correlates with the upregulation of the $\mathrm{Wnt} / \beta$-catenin signaling pathway and the poor survival of gastric cancer patients. Int J Mol Med 34: 197-204, 2014.

4. Oo HZ, Sentani K, Sakamoto N, Anami K, Naito Y, Uraoka N, Oshima T, Yanagihara K, Oue N and Yasui W: Overexpression of ZDHHC14 promotes migration and invasion of scirrhous type gastric cancer. Oncol Rep 32: 403-410, 2014.

5. Lu F, Xue JX, Hu YC, Gan L, Shi Y, Yang HS and Wei YQ: CARP is a potential tumor suppressor in gastric carcinoma and a single-nucleotide polymorphism in CARP gene might increase the risk of gastric carcinoma. PLoS One 9: e97743, 2014.

6. Li Z, Chang X, Dai D, Deng P and Sun Q: RASSF10 is an epigenetically silenced tumor suppressor in gastric cancer. Oncol Rep 31: 1661-1668, 2014.

7. Legrand D: Lactoferrin, a key molecule in immune and inflammatory processes. Biochem Cell Biol 90: 252-268, 2012.

8. Legrand D, Pierce A, Elass E, Carpentier M, Mariller C and Mazurier J: Lactoferrin structure and functions. Adv Exp Med Biol 606: 163-194, 2008.

9. Gibbons JA, Kanwar RK and Kanwar JR: Lactoferrin and cancer in different cancer models. Front Biosci (Schol Ed) 3: 1080-1088, 2011.

10. Bezault J, Bhimani R, Wiprovnick J and Furmanski P: Human lactoferrin inhibits growth of solid tumors and development of experimental metastases in mice. Cancer Res 54: 2310-2312, 1994.

11. Varadhachary A, Wolf JS, Petrak K, O'Malley BW Jr, Spadaro M, Curcio C, Forni G and Pericle F: Oral lactoferrin inhibits growth of established tumors and potentiates conventional chemotherapy. Int J Cancer 111: 398-403, 2004.

12. Tsuda H, Sekine K, Nakamura J, Ushida Y, Kuhara T, et al: Inhibition of azoxymethane initiated colon tumor and aberrant crypt foci development by bovine lactoferrin administration in F344 rats. Adv Exp Med Biol 443: 273-284, 1998.

13. Sekine K, Watanabe E, Nakamura J, Takasuka N, et al: Inhibition of azoxymethane-initiated colon tumor by bovine lactoferrin administration in F344 rats. Jpn J Cancer Res 88: 523-526, 1997.

14. Matsuda Y, Saoo K, Hosokawa K, Yamakawa K, Yokohira M, Zeng Y, Takeuchi H and Imaida K: Post-initiation chemopreventive effects of dietary bovine lactoferrin on 4-(methylnitrosamino)-1(3-pyridyl)-1-butanone-induced lung tumorigenesis in female $\mathrm{A} / \mathrm{J}$ mice. Cancer Lett 246: 41-46, 2007.

15. Li WY, Li QW, Han ZS, Jiang ZL, Yang H, Li J and Zhang XB: Growth suppression effects of recombinant adenovirus expressing human lactoferrin on cervical cancer in vitro and in vivo. Cancer Biother Radiopharm 26: 477-483, 2011.
16. Nakajima M, Shinoda I, Samejima Y, Miyauchi H, Fukuwatari Y and Hayasawa $\mathrm{H}$ : Lactoferrin as a suppressor of cell migration of gastrointestinal cell lines. J Cell Physiol 170: 101-105, 1997.

17. Zhou Y, Zeng Z, Zhang W, Xiong W, Wu M, Tan Y, Yi W, Xiao L, Li X, Huang C, et al: Lactotransferrin: a candidate tumor suppressor - Deficient expression in human nasopharyngeal carcinoma and inhibition of NPC cell proliferation by modulating the mitogen-activated protein kinase pathway. Int J Cancer 123: 2065-2072, 2008.

18. Livak KJ and Schmittgen TD: Analysis of relative gene expression data using real-time quantitative PCR and the 2(-Delta Delta C(T)) Method. Methods 25: 402-408, 2001.

19. Pfaffl MW: A new mathematical model for relative quantification in real-time RT-PCR. Nucleic Acids Res 29: e45, 2001.

20. Deng M, Zhang W, Tang H, Ye Q, Liao Q, Zhou Y, Wu M, Xiong W, Zheng Y, Guo X, et al: Lactotransferrin acts as a tumor suppressor in nasopharyngeal carcinoma by repressing AKT through multiple mechanisms. Oncogene 32: 4273-4283, 2013.

21. Ward PP, Paz E and Conneely OM: Multifunctional roles of lactoferrin: a critical overview. Cell Mol Life Sci 62: 2540-2548, 2005.

22. Rodrigues L, Teixeira J, Schmitt F, Paulsson M and Månsson HL: Lactoferrin and cancer disease prevention. Crit Rev Food Sci Nutr 49: 203-217, 2009.

23. Shaheduzzaman S, Vishwanath A, Furusato B, Cullen J, Chen Y, Bañez L, Nau M, Ravindranath L, Kim KH, Mohammed A, et al: Silencing of Lactotransferrin expression by methylation in prostate cancer progression. Cancer Biol Ther 6: 1088-1095, 2007.

24. Iijima H, Tomizawa Y, Iwasaki Y, Sato K, Sunaga N, Dobashi K, Saito R, Nakajima T, Minna JD and Mori M: Genetic and epigenetic inactivation of LTF gene at 3p21.3 in lung cancers. Int J Cancer 118: 797-801, 2006.

25. Yi HM, Li H, Peng D, Zhang HJ, Wang L, Zhao M, Yao KT and Ren CP: Genetic and epigenetic alterations of LTF at 3p21.3 in nasopharyngeal carcinoma. Oncol Res 16: 261-272, 2006.

26. Campbell T, Skilton RA, Coombes RC, Shousha S, Graham MD and Luqmani YA: Isolation of a lactoferrin cDNA clone and its expression in human breast cancer. Br J Cancer 65: 19-26, 1992.

27. Kholodnyuk ID, Kozireva S, Kost-Alimova M, Kashuba V, Klein $\mathrm{G}$ and Imreh S: Down regulation of $3 p$ genes, LTF, SLC38A3 and DRR1, upon growth of human chromosome 3 -mouse fibrosarcoma hybrids in severe combined immunodeficiency mice. Int J Cancer 119: 99-107, 2006.

28. Yang Y, Li J, Szeles A, Imreh MP, Kost-Alimova M, Kiss H, Kholodnyuk I, Fedorova L, Darai E, Klein G and Imreh S: Consistent downregulation of human lactoferrin gene, in the common eliminated region 1 on $3 p 21.3$, following tumor growth in severe combined immunodeficient (SCID) mice. Cancer Lett 191: 155-164, 2003.

29. Huang P, Han J and Hui L: MAPK signaling in inflammation-associated cancer development. Protein Cell 1: 218-226, 2010.

30. Wagner EF and Nebreda AR: Signal integration by JNK and p38 MAPK pathways in cancer development. Nat Rev Cancer 9: 537-549, 2009.

31. You H, Lei P and Andreadis ST: JNK is a novel regulator of intercellular adhesion. Tissue Barriers 1: e26845, 2013.

32. Yuan F, Xu Z, Yang M, Wei Q, Zhang Y, Yu J, Zhi Y, Liu Y, Chen $Z$ and Yang J: Overexpressed DNA polymerase iota regulated by JNK/c-Jun contributes to hypermutagenesis in bladder cancer. PLoS One 8: e69317, 2013.

33. Okada M, Shibuya K, Sato A, Seino S, Watanabe E, Suzuki S, Seino $M$ and Kitanaka C: Specific role of JNK in the maintenance of the tumor-initiating capacity of A549 human non-small cell lung cancer cells. Oncol Rep 30: 1957-1964, 2013.

34. Suzuki K and Matsubara H: Recent advances in $\mathrm{p} 53$ research and cancer treatment. J Biomed Biotechnol 2011: 978312, 2011.

35. Jiang L, Chang J, Zhang Q, Sun L and Qiu X: MicroRNA hsa-miR-125a-3p activates p53 and induces apoptosis in lung cancer cells. Cancer Invest 31: 538-544, 2013.

36. Marcel V, Ghayad SE, Belin S, Therizols G, Morel AP, Solano-Gonzàlez E, Vendrell JA, Hacot S, Mertani HC, Albaret MA, et al: p53 acts as a safeguard of translational control by regulating fibrillarin and rRNA methylation in cancer. Cancer Cell 24: 318-330, 2013.

37. Wang Y, Zhang YX, Kong CZ, Zhang Z and Zhu YY: Loss of P53 facilitates invasion and metastasis of prostate cancer cells. Mol Cell Biochem 384: 121-127, 2013. 